\title{
PORTADORES ASINTOMÁTICOS DE Staphylococcus aureus EN TRABAJADORES DEL HOSPITAL REGIONAL DE ICA, PERÚ 2011
}

\author{
Rocío Córdova-Vicerrel ${ }^{1,2, a}$, Patricia Cavero-Trigozo ${ }^{1,3, b}$, \\ Julissa Huaranga-Bravo ${ }^{1, \mathrm{~b}}$, Carlos Pachas-Canales ${ }^{1, \mathrm{~b}}$
}

1. Facultad de Medicina, Universidad Nacional San Luis Gonzaga. Ica, Perú.

2. Hospital Regional de Ica. Perú

3. Sociedad Científica de Estudiantes de Medicina de Ica.

a. Biólogo b. Estudiante de Medicina

\section{RESUMEN}

Objetivo: Determinar la prevalencia de portadores asintomáticos y sensibilidad antimicrobiana de Staphylococcus aureus en trabajadores del Hospital Regional de Ica. Material y métodos: Estudio de tipo descriptivo transversal realizado en noviembre del 2011. Según muestreo aleatorio simple sin reposición, la muestra fue de 131 trabajadores. Se obtuvieron los datos con la aplicación de una ficha y la extracción de las muestras mediante hisopado, obteniendo 262 cepas de manos y fosas nasales. El aislamiento, identificación y pruebas de sensibilidad se efectuaron según los criterios del Clinical and Laboratory Standars Institute (CLSI). Los datos se procesaron en el programa SPSS, realizando análisis univariado y pruebas de significación estadística. Resultados: La prevalencia de portadores asintomáticos fue de $12,98 \%$ en total, $10,7 \%$ en manos y $5,3 \%$ en fosas nasales. Todos los gérmenes fueron sensibles a: meticilina, cefaclor, ceftazidima, vancomicina y rifampicina. Sólo fueron resistentes algunas cepas de fosas nasales $(7,1 \%)$ a: oxacilina, dicloxacilina, claritromicina y cloranfenicol. Conclusiones: La prevalencia de portadores asintomáticos de Staphylococcus aureus fue baja. Se observó menor frecuencia de portadores cuando se utilizaban algunas medidas de protección. La resistencia antimicrobiana también fue baja. Se debe realizar un estudio caso-control para identificar los factores de protección. (Rev. méd. panacea 2011; 1:59-66).

Palabras Clave: Staphylococcus aureus, portador asintomático, sensibilidad antimicrobiana, trabajadores de salud. (fuente: DeCS BIREME)

\section{Staphylococcus aureus ASYMPTOMATIC CARRIERS IN WORKERS FROM ICA REGIONAL HOSPITAL, PERU 2011.}

\begin{abstract}
Objective: To determinate the prevalence of asymptomatic carriers and antimicrobial susceptibility of Staphylococcus aureus in workers at Ica Regional Hospital. Material and Methods: A descriptive-transversal study realized in November 2011. A simple aleatory sampling without repositioning, a total of 131 workers participated in the study. Dates were collected using a file and the samples by cottons sawbs, obtaining 262 nasal and hand strains. The isolation, identification and susceptibility testing were made using the criteria of Clinical and Laboratory Standars Institute (CLSI). Data were processed in the SPSS version 18, doing univariate analysis and statistical significance tests. Results: The prevalence of asymptomatic carriers was $12.98 \%$, in which $5.3 \%$ is the rate of nasal carriage and $10.7 \%$ in hands. All isolates were susceptible to: methicillin, cefaclor, ceftazidime, vancomycin and rifampin. Only a few nasal isolates were resistant $(7.1 \%)$ to: oxacillin, dicloxacilin, clarithromycin and chloramphenicol. Conclusion: The prevalence of asymptomatic carriers was low. It was showed a lower carrier frequency when workers used protective measures $(p>0.05)$. The antimicrobial resistance was low, too. There must be a casecontrol study to identify protective factors. (Rev. méd. panacea 2011; 1:59 -66).
\end{abstract}

Key words: Staphylococcus aureus, asymptomatic carriers, antimicrobial resistance, health workers. (source: MeSH NLM).

\section{INTRODUCCIÓN}

Staphylococcus aureus (S. aureus) son microorganismos Gram positivos, que, en determinadas condiciones pueden ser causantes de diversas infecciones en el ser humano, éstas van desde una simple infección cutánea hasta manifestaciones sistémicas que pueden llevar a la muerte por sepsis. Se caracteriza por producir coagulación de la fibrina plasmática, siendo la prueba de la coagulasa determinante para su identificación ${ }^{(1)}$.
Poseen una variedad de elementos que le confieren características propias a su patogenicidad y protección ante los mecanismos de defensa del hospedero. Por otro lado, considerando su material genético, el gen mec A constituye uno de los determinantes de resistencia a Meticilina ${ }^{(2,3)}$, mientras que el gen erm lo es para Clindamicina y Eritromicina ${ }^{(3,4)}$

Esta bacteria conforma la flora residente, generando un estado de portador en manos, periné y nariz, reportándose 
una mayor prevalencia en esta última ${ }^{(5,6,7)}$. Debido a que la contaminación es de persona a persona el personal de salud se encuentra expuesto, pudiendo constituir un reservorio importante que repercute sobre los pacientes y éstos sobre la comunidad ${ }^{(6,8)}$.

La prevalencia internacional de infecciones intrahospitalarias, por S. aureus fluctúa entre 15,5 y $82,8 \%$ ${ }^{(9-12)}$, encontrándose la nacional entre $9,7-24,2 \%^{(4,13,14)}$.

La prevalencia de portación nasal, en trabajadores asistenciales, encontrada en la literatura latinoamericana fluctúa entre 10,5 y $28,6 \%{ }^{(7,15-17)}$. Por otro lado, Guzmán ${ }^{(18)}$ afirma que las infecciones intrahospitalarias por $S$. aureus meticilino resistente han sobrepasado el $50 \%$ de prevalencia en la mayoría de los países de Latinoamérica y, el número de infecciones adquiridas en la comunidad está en aumento.

Conocida la importancia del S. aureus como agente causal de infecciones intrahospitalarias, fue motivación suficiente para realizar el presente estudio, que tuvo como objetivo: determinar la prevalencia de portadores asintomáticos y sensibilidad antimicrobiana de $S$. aureus en trabajadores del Hospital Regional de Ica.

\section{MATERIAL Y MÉTODOS}

Muestreo: Para este estudio fueron considerados todos los trabajadores de la institución, en total 589. Empleando la relación oficial se tomó una muestra aleatoria de 120 individuos, lo que significa un nivel de confianza del $99 \%$, para una prevalencia esperada del $35 \%$ y un peor resultado de $25 \%$, con un error probable de $0,1 \%$. A esto se le agregó once sujetos por la posible pérdida de muestra. En total se evaluó a 131 trabajadores entre asistenciales y no asistenciales, durante el mes de octubre del 2011.

La asignación de trabajadores para la muestra se realizó mediante la lista general, que contenía: nombre y apellido, cargo y área de trabajo. Con el listado se asignó un número a cada uno de ellos, seleccionándolos luego, mediante un muestreo aleatorio simple sin reposición. Cuando el trabajador no deseaba participar en la investigación, se realizaba un nuevo sorteo para seleccionar otro. Fueron excluidos todos los trabajadores que habían presentado una infección en las últimas dos semanas o si habían recibido antibióticos.

Toma de Muestra: La muestra de fosas nasales fue tomada con un hisopo estéril humedecido en solución salina fisiológica, realizando tres movimientos rotatorios enérgicos en sentido horario en las narinas anteriores y regresándolo al mismo tubo de ensayo, para su transporte y posterior siembra. La muestra de manos, se tomó con otro hisopo de las mismas características, el cual fue friccionado en los pliegues de la palma y dorso de la mano, en el lecho ungueal y en los espacios interdigitales ${ }^{(17,19,20)}$.

Cultivo: Las muestras tomadas fueron transportadas al laboratorio de Microbiología de la Facultad de Medicina de la Universidad Nacional San Luis Gonzaga, en un lapso de tiempo no mayor a dos horas, allí fueron sembradas en el medio agar manitol hipertónico aplicando la técnica de agotamiento y estría. Luego fueron incubadas a una temperatura de $37^{\circ} \mathrm{C}$, durante 24 horas, en un medio aerobio. Los cultivos que no presentaron crecimiento de colonias fueron incubados hasta 48 horas, antes de ser dados como negativos.

Coloración Gram: Luego de crecidas las colonias, se realizó la identificación, de acuerdo a las características macroscópicas, en típicas o atípicas (considerando una cepa patrón proporcionada por la Facultad de Ciencias de la Universidad Nacional San Luis Gonzaga). Posteriormente, se seleccionó una de las colonias y se extendió en láminas portaobjetos, tiñéndolas mediante la técnica de coloración de Gram; catalogándolas luego en bacterias Gram positivas y Gram negativas.

Identificación Bioquímica: Luego de la tinción, a todas las muestras se les realizó la prueba de la catalasa, identificándose como perteneciente al género Staphylococcus a los cocos Gram positivos que formaron burbujas (reacción con el peróxido de hidrógeno). Las cepas que no presentaron este hallazgo no fueron consideradas para las siguientes pruebas bioquímicas.

Todas las cepas determinadas como catalasa positivas fueron sometidas a la prueba de la coagulasa, para determinar la especie $S$. aureus; y hemolisina como confirmación de la identificación de esta especie.

Pruebas de sensibilidad: A las cepas aisladas de S. aureus se les aplicó las pruebas de sensibilidad en el medio Müller-Hinton, utilizando el método de Kirby-Bauer con escala de Mac Farland de 0,5 y siguiendo las pautas establecidas por el Clinical and Laboratory Standars Institute (CLSI).

Control de Calidad: Como se dijo anteriormente, para la comparación se utilizó una cepa patrón estandarizada de $S$. aureus obtenida en el laboratorio de Microbiología de la Facultad de Ciencias, procedente del Instituto Nacional de Salud (INS-27522).

Además de los datos obtenidos con las pruebas microbiológicas y sensibilidad, se tomaron en cuenta otros 




Figura 1. Portadores asintomáticos de Staphylococcus aureus en trabajadores del Hospital Regional de Ica según proceso de análisis en el laboratorio.

datos como: filiación (nombres, apellidos, edad, sexo), cargo, área de servicio, grado de exposición (según contacto con pacientes), tiempo de trabajo (en el hospital, área y turno), última infección presentada (tiempo, zona y tratamiento), administración de antibióticos (tipo y tiempo), administración de corticoides (tipo y tiempo), uso de métodos de barrera (guantes, mascarillas, mandil, gorro, anteojos y botas); práctica del lavado de manos (antes y después de atender a pacientes, o de realizar algún procedimiento), manipulación de instrumentos (esterilizados o no), atención de pacientes (promedio de atendidos y tiempo de atención) y limpieza del área de trabajo (por día y semana).

Análisis: Con la información obtenida se creó una base de datos utilizando la versión 18 del Programa SPSS, con el que se procesaron los datos y obtuvieron para el análisis univariado: medias, frecuencias, porcentajes y prevalencias; posteriormente se aplicaron pruebas de significación como: Chi cuadrado, prueba exacta de Fisher y t-Student,. Se consideró significativa cuando el valor fue $p<0,05$.

\section{RESULTADOS}

En total fueron 131 trabajadores de la institución entre asistenciales y no asistenciales. Se cultivaron en total 262 muestras: 131 de manos y 131 de fosas nasales. En el agar manitol hipertónico, de las muestras de manos, crecieron 124 cepas formadoras de colonias y; de las muestras de fosas nasales, sólo 125 de las sembradas. Tras el extendido en la lámina portaobjeto, la coloración Gram mostró 35 Gram positivos y 214 Gram negativos. A todas las muestras se les practicó la prueba catalasa que diferencia al género Staphylococcus (Figura 1).

De los Gram positivos: 22 fueron catalasa positivo y trece negativo. Mientras que de los Gram negativos: tres fueron catalasa positivo y 211 negativo. A los catalasa positivos, se les aplicó la prueba coagulasa para identificar la especie $S$. aureus, resultando todas las cepas coagulasa positivas. Posteriormente fueron sometidas a la prueba de hemólisis, confirmándose catorce cepas de $S$. aureus en manos y siete en fosas nasales (Figura 1).

Del total de la muestra: $13 \%$ fueron médicos, $34,4 \%$ enfermeros, $17,6 \%$ administrativos, $12,2 \%$ artesanos; $10,7 \%$ químicos farmacéuticos, $2,3 \%$ obstetras, $8,4 \%$ técnicos en nutrición, $1,5 \%$ cirujanos-dentistas, $5,3 \%$ técnicos de laboratorio; también fueron incluidos un nutricionista, dos psicólogos, un biólogo y otros trabajadores. La edad fluctuó entre $45,79 \pm 10.0$ años; 89 $(67,9 \%)$ fueron de sexo femenino.

En general, el empleo de instrumentos esterilizados en los diferentes servicios, mostró una menor prevalencia de portadores $(3,1 \%)$ frente a los trabajadores que no manipulaban instrumentos con dichas características. Los 
trabajadores que se lavaban las manos antes y después de atender a los pacientes, presentaron una menor prevalencia de portador asintomático (1,5\%), en comparación con los que no adoptaban esta medida de protección, estos fueron pocos trabajadores.

La prevalencia de portadores asintomáticos de Staphylococcus aureus en manos fue de 10,7 \%. Con el uso de barreras de protección (como: guantes, mascarilla, mandiles, lentes, gorro y botas) se observó una menor prevalencia de portador en manos; sin embargo, las diferencias tampoco fueron significativas (Tabla 1).

En portadores de manos hubo diferencias entre los grupos etáreos, orientadas a una mayor prevalencia a medida que aumentaba la edad; esto tampoco fue significativo. Los trabajadores que se lavaban las manos con una frecuencia menor a cinco veces por turno, presentaron más casos $(12,7 \%)$ de estado de portador en relación a los que lo hacían entre 6 y 9 veces $(5,4 \%)$. No se encontró una relación entre el uso de guantes y portador (cuando se usaron menos de cinco pares de guantes por día la prevalencia fue de $10,8 \%$, frente a cuando se utilizaron entre 6 y 9 que fue de 18,8\%) (Tabla 1).

Los mayores porcentajes de portadores en manos, se encontraron entre los que: no utilizaban mascarilla, mandiles, lentes, gorro y botas $(13,6 \%, 8,4 \%, 11,3 \%, 11,5 \%$ y $11,6 \%$ respectivamente). Esto no sucede en los portadores nasales, salvo con las mascarillas.

La prevalencia del estado de portador asintomático de $S$. aureus, en fosas nasales, fue de 5,3\%. La edad de mayor prevalencia fue entre 48 y 59 años $(9,4 \%)$. En relación al grado de exposición no se observaron diferencias.

La frecuencia del lavado de manos fue inversamente proporcional a la prevalencia de portadores asintomáticos en fosas nasales, encontrándose porcentajes de 7,5\% y $3,2 \%$ entre los trabajadores cuya higiene de manos fue menor de cinco y mayor de diez veces al día, respectivamente. Por otro lado, la mayor prevalencia de portadores se observó entre los que usaban menos de 10 pares de guantes al día (Tabla 2).

Las Tablas 3 y 4 muestran los resultados de sensibilidad en manos y fosas nasales. Las cepas de manos presentaron sensibilidad: $100 \%$ a meticilina y vancomicina, 92,9\% a oxacilina, dicloxacilina, cefaclor y ceftazidima y $85,7 \%$ a claritromicina, rifampicina y cloranfenicol; mientras que, la resistencia fue de: $7,1 \%$ a oxacilina, dicloxacilina, claritromicina y cloranfenicol. Con respecto a fosas nasales, se encontró sensibilidad: $100 \%$ a meticilina, oxacilina, dicloxacilina, claritromicina, cefaclor, ceftazidima, vancomicina y rifampicina y $85,7 \%$ a cloranfenicol; no se observó resistencia a ningún fármaco.

Tabla 1. Portadores asintomáticos de Staphylococcus aureus en trabajadores del Hospital Regional de Ica según lugar de extracción: Mano.

\begin{tabular}{|c|c|c|c|c|c|}
\hline \multirow{2}{*}{ Variables } & \multicolumn{2}{|c|}{ Portador } & \multicolumn{2}{|c|}{ No Portador } & \multirow{2}{*}{$p$} \\
\hline & $n=14$ & $(\%)$ & $n=117$ & $(\%)$ & \\
\hline \multicolumn{6}{|l|}{ Sexo } \\
\hline Hombre & 4 & (9.5) & 38 & $(90.5)$ & 0,77 \\
\hline Mujer & 10 & $(11.2)$ & 79 & $(99.8)$ & \\
\hline \multicolumn{6}{|l|}{ Edad } \\
\hline $24-35$ & 1 & $(4.2)$ & 23 & $(95.8)$ & 0,48 \\
\hline $36-47$ & 4 & (9.1) & 40 & $(90,9)$ & \\
\hline $48-59$ & 7 & $(13,2)$ & 46 & $(86,8)$ & \\
\hline $60-69$ & 2 & $(20,0)$ & 8 & $(80,0)$ & \\
\hline \multicolumn{6}{|c|}{ Exposición con pacientes } \\
\hline Alto & 7 & $(9,3)$ & 68 & $(90,7)$ & 0,56 \\
\hline Bajo & 7 & $(12,5)$ & 49 & $(87,5)$ & \\
\hline \multicolumn{6}{|c|}{ Tiempo de servicio } \\
\hline$\leq 200$ meses & 5 & $(7,2)$ & 64 & $(92,8)$ & 0,91 \\
\hline$>200$ meses & 9 & $(17,3)$ & 53 & $(82,7)$ & \\
\hline \multicolumn{6}{|c|}{ Uso de mascarilla } \\
\hline $\mathrm{Si}$ & 3 & $(6,0)$ & 47 & $(94,0)$ & 0,17 \\
\hline No & 11 & $(13,6)$ & 70 & $(86,4)$ & \\
\hline \multicolumn{6}{|l|}{ Uso de mandil } \\
\hline $\mathrm{Si}$ & 3 & $(2,3)$ & 40 & $(30,5)$ & 0,34 \\
\hline No & 11 & $(8,4)$ & 77 & $(58,8)$ & \\
\hline \multicolumn{6}{|l|}{ Uso de lentes } \\
\hline $\mathrm{Si}$ & 0 & $(0,0)$ & 7 & $(100,0)$ & 0,88 \\
\hline No & 14 & $(11,3)$ & 110 & $(88,7)$ & \\
\hline \multicolumn{6}{|l|}{ Uso de gorros } \\
\hline $\mathrm{Si}$ & 1 & $(5,6)$ & 17 & $(94,4)$ & 0,45 \\
\hline No & 13 & $(11,5)$ & 100 & $(88,5)$ & \\
\hline \multicolumn{6}{|l|}{ Uso de botas } \\
\hline $\mathrm{Si}$ & 0 & $(0,0)$ & 10 & $(100,0)$ & 0,26 \\
\hline No & 14 & $(11,6)$ & 107 & $(88,4)$ & \\
\hline \multicolumn{6}{|c|}{ Lavado de manos por turno } \\
\hline$<5$ & 8 & $(12,7)$ & 55 & $(87,3)$ & 0,47 \\
\hline 6 a 9 & 2 & $(5,4)$ & 35 & $(94,6)$ & \\
\hline$>10$ & 4 & $(12,9)$ & 27 & $(87,1)$ & \\
\hline \multicolumn{6}{|c|}{ Uso de guantes (pares) } \\
\hline$<5$ & 11 & $(10,8)$ & 91 & $(89,2)$ & 0,27 \\
\hline 6 a 9 & 3 & $(18,8)$ & 13 & $(81,2)$ & \\
\hline$>10$ & 0 & $(0,0)$ & 13 & $(100,0)$ & \\
\hline
\end{tabular}


Tabla 2. Portadores asintomáticos de Staphylococcus aureus en trabajadores del Hospital Regional de Ica según lugar de extracción: Fosas nasales.

\begin{tabular}{|c|c|c|c|c|c|}
\hline \multirow{2}{*}{ Variables } & \multicolumn{2}{|c|}{ Portador } & \multicolumn{2}{|c|}{ No Portador } & \multirow{2}{*}{$p$} \\
\hline & $\mathrm{n}=7$ & $(\%)$ & $n=124$ & $(\%)$ & \\
\hline \multicolumn{6}{|l|}{ Sexo } \\
\hline Hombre & 1 & $(2,4)$ & 41 & $(97,6)$ & 0,30 \\
\hline Mujer & 6 & $(6,7)$ & 83 & $(93,3)$ & \\
\hline \multicolumn{6}{|l|}{ Edad } \\
\hline $24-35$ & 1 & $(4,2)$ & 23 & $(95,8)$ & 0,36 \\
\hline $36-47$ & 1 & $(2,3)$ & 43 & $(97,7)$ & \\
\hline $48-59$ & 5 & $(9,4)$ & 48 & $(90,6)$ & \\
\hline $60-69$ & 0 & $(0,0)$ & 10 & $(100)$ & \\
\hline \multicolumn{6}{|c|}{ Exposición con pacientes } \\
\hline Alto & 4 & $(5,3)$ & 71 & $(94.7)$ & 0,99 \\
\hline Bajo & 3 & $(5,4)$ & 53 & $(94,6)$ & \\
\hline \multicolumn{6}{|l|}{ Tiempo de servicio } \\
\hline$\leq 200$ meses & 4 & $(5,8)$ & 65 & $(94,2)$ & 0,81 \\
\hline$>200$ meses & 3 & $(4,8)$ & 59 & $(95,2)$ & \\
\hline \multicolumn{6}{|l|}{ Uso de mascarilla } \\
\hline $\mathrm{Si}$ & 2 & $(4,0)$ & 48 & $(96,0)$ & 0,59 \\
\hline No & 5 & $(6,2)$ & 76 & $(93,8)$ & \\
\hline \multicolumn{6}{|l|}{ Uso de mandil } \\
\hline $\mathrm{Si}$ & 3 & $(7,0)$ & 40 & $(93,0)$ & 0,56 \\
\hline No & 4 & $(4,5)$ & 84 & $(95,5)$ & \\
\hline \multicolumn{6}{|l|}{ Uso de lentes } \\
\hline $\mathrm{Si}$ & 1 & $(14,3)$ & 6 & $(85,7)$ & 0,28 \\
\hline No & 6 & $(4,8)$ & 118 & $(95,2)$ & \\
\hline \multicolumn{6}{|l|}{ Uso de gorros } \\
\hline $\mathrm{Si}$ & 1 & $(5,6)$ & 17 & $(94,4)$ & 0,97 \\
\hline No & 6 & $(5,3)$ & 107 & $(94,7)$ & \\
\hline \multicolumn{6}{|l|}{ Uso de botas } \\
\hline $\mathrm{Si}$ & 1 & $(10.0)$ & 9 & $(90,0)$ & 0,50 \\
\hline No & 6 & $(5,0)$ & 115 & $(95,0)$ & \\
\hline \multicolumn{6}{|c|}{ Lavado de manos por turnos } \\
\hline$<5$ & 4 & $(7,5)$ & 59 & $(92,5)$ & 0,82 \\
\hline 6 a 9 & 2 & $(5,4)$ & 35 & $(94,6)$ & \\
\hline$>10$ & 1 & $(3,2)$ & 30 & $(96,8)$ & \\
\hline \multicolumn{6}{|c|}{ Uso de guantes (pares) } \\
\hline$<5$ & 6 & $(5,9)$ & 96 & $(94,1)$ & 0,66 \\
\hline 6 a 9 & 1 & $(6,3)$ & 15 & $(93,7)$ & \\
\hline$>10$ & 0 & $(0,0)$ & 13 & $(100)$ & \\
\hline
\end{tabular}

\section{DISCUSIÓN}

La prevalencia de portadores asintomáticos de $S$. aureus, entre los trabajadores del Hospital Regional de Ica, es de $12,98 \%$ (siendo $10,7 \%$ en manos y $5,3 \%$ en fosas nasales); ésta es menor que la hallada por Zelaya y col ${ }^{(19)}$, quienes encontraron $46,7 \%$ en manos y $68,7 \%$ en fosas nasales.
La baja prevalencia encontrada, podría deberse a diversos factores, como el mencionado por Platzer ${ }^{(21)}$ que observó cierto antagonismo entre el crecimiento de $S$. aureus y Difteroides, asumiendo que éste producía cambios en el nicho ecológico del Staphylococcus. En nuestro caso no se hizo la identificación de Difteroides, este pudo haber influido sobre el menor crecimiento de $S$. aureus. Por otro lado, el uso de medios de enriquecimiento antes de la siembra en el medio selectivo, podría haber incrementado su crecimiento ${ }^{(22)}$, lo cual tampoco se realizó.

Otros factores que podrían explicar esta baja prevalencia, pese a la precaria infraestructura en la que actualmente funciona el hospital, sería el uso de alcohol glicerinado ${ }^{(23)}$, ya que la mayoría de los servicios cuentan con un dispensador de éste producto; el cual parece tener una alta potencialidad antimicrobiana ${ }^{(24)}$. El lavado de manos es un factor protector reconocido por varios investigadores ${ }^{\text {(22, 24- }}$ 26), pero, en nuestro caso, no encontramos diferencias significativas entre los que se lavaban más o menos veces para ser o no portador.

Puesto que dicha bacteria se encuentra en objetos inanimados como: camillas, mesas, cunas y balanzas ${ }^{(27)}$, no podemos considerar que la limpieza y desinfección de los ambientes e instrumentos haya contribuido a dicho hallazgo, ya que no se presentaron diferencias con respecto a ser portador según este factor. Asimismo se debe considerar que el uso de desinfectantes químicos, para la limpieza de superficies, parece disminuir la contaminación de microorganismos ${ }^{(28)}$.

Los resultados discutidos antes nos muestran una serie de vacios, por lo que consideramos necesario realizar una investigación caso control para identificar los probables factores de protección.

Nuestros resultados no mostraron resistencia a meticilina, a diferencia de lo hallado por Mendoza ${ }^{(20)}$, que encontró un $20 \%$ de resistencia en fosas nasales. Zelaya y col. ${ }^{(19)}$ obtuvieron una sensibilidad cercana al $100 \%$ para oxacilina, siendo sus resultados similares a los nuestros. La sensibilidad del $100 \%$ a Vancomicina concuerda con la reportada por Paz, Mamani y Montalvo (13, 29, 33), contrastando con los resultados de Zelaya ${ }^{(19)}$. La resistencia a claritromicina y cloranfenicol, encontrada por nosotros, fue de $7,1 \%$, lo que difiere con lo publicado por Montalvo ${ }^{(33)}$. Esta baja prevalencia de cepas resistentes podría deberse a una adecuada prescripción y uso racional de antimicrobianos por parte del personal del hospital ${ }^{(34)}$. Además, debemos considerar que esto evita gastos innecesarios, pues, como se sabe, el costo del tratamiento de $S$. aureus meticilino resistente se quintuplica con respecto a $S$. aureus meticilino sensible ${ }^{(9)}$. 
Tabla 3. Portadores asintomáticos de Staphylococcus aureus en trabajadores del Hospital Regional de Ica según sensibilidad antimicrobiana en mano, 2011.

\begin{tabular}{lcccccc}
\hline \multirow{2}{*}{ Antimicrobiano } & \multicolumn{2}{c}{ Sensible } & \multicolumn{2}{c}{ Intermedio } & \multicolumn{2}{c}{ Resistente } \\
& $\mathbf{n}$ & $\mathbf{( \% )}$ & $\mathbf{n}$ & $\mathbf{( \% )}$ & $\mathbf{n}$ & $(\%)$ \\
\hline Meticilina & 14 & $(100)$ & 0 & $(0)$ & 0 & $(0)$ \\
Oxacilina & 13 & $(92,9)$ & 0 & $(0)$ & 1 & $(7,1)$ \\
Dicloxacilina & 13 & $(92,9)$ & 0 & $(0)$ & 1 & $(7,1)$ \\
Claritromicina & 12 & $(85,7)$ & 1 & $(7,1)$ & 1 & $(7,1)$ \\
Cefaclor & 13 & $(92,9)$ & 1 & $(7,1)$ & 0 & $(0)$ \\
Ceftazidima & 12 & $(92,9)$ & 2 & $(14,3)$ & 0 & $(0)$ \\
Vancomicina & 14 & $(100)$ & 0 & $(0)$ & 0 & $(0)$ \\
Rifampicina & 12 & $(85,7)$ & 2 & $(14,3)$ & 0 & $(0)$ \\
Cloranfenicol & 12 & $(85,7)$ & 1 & $(7,1)$ & 1 & $(7,1)$ \\
\hline
\end{tabular}

Tabla 4. Portadores asintomáticos de Staphylococcus aureus en trabajadores del Hospital Regional de Ica según sensibilidad antimicrobiana en fosas nasales, 2011.

\begin{tabular}{lcccccc}
\hline \multirow{2}{*}{ Antimicrobiano } & \multicolumn{2}{c}{ Sensible } & \multicolumn{2}{c}{ Intermedio } & \multicolumn{2}{c}{ Resistente } \\
& $\mathbf{n}$ & $\mathbf{( \% )}$ & $\mathbf{n}$ & $\mathbf{( \% )}$ & $\mathbf{n}$ & $\mathbf{( \% )}$ \\
\hline Meticilina & 7 & $(100)$ & 0 & $(0)$ & 0 & $(0)$ \\
Oxacilina & 7 & $(100)$ & 0 & $(0)$ & 0 & $(0)$ \\
Dicloxacilina & 7 & $(100)$ & 0 & $(0)$ & 0 & $(0)$ \\
Claritromicina & 7 & $(100)$ & 0 & $(0)$ & 0 & $(0)$ \\
Cefaclor & 7 & $(100)$ & 0 & $(0)$ & 0 & $(0)$ \\
Ceftazidima & 7 & $(100)$ & 0 & $(0)$ & 0 & $(0)$ \\
Vancomicina & 7 & $(100)$ & 0 & $(0)$ & 0 & $(0)$ \\
Rifampicina & 7 & $(100)$ & 0 & $(0)$ & 0 & $(0)$ \\
Cloranfenicol & 6 & $(85,7)$ & 1 & $(16,3)$ & 0 & $(0)$ \\
\hline
\end{tabular}

Otro problema a tener en cuenta es: ¿Qué hacer con los portadores, sobre todo del germen resistente?, felizmente no es nuestro caso, dado el bajo nivel de resistencia que tenemos. Diversos trabajos tratan el tema de la erradicación, es relevante un informe COCHRANE ${ }^{(35)}$ en el que se dice que los antisépticos tópicos, antisépticos detergentes y fármacos antimicrobianos sistémicos usados como monoterapia o terapia combinada no serían eficaces para la erradicación de $S$. aureus meticilino resistente. Por lo tanto, en estos casos sería útil el uso adecuado de las barreras (guantes y mascarillas) para evitar la diseminación.
Investigadores nacionales ${ }^{(1,4,33,36,37)}$ e internacionales ${ }^{(38)}$ sugieren el empleo de la oxacilina para sustituir el resultado del test de sensibilidad a meticilina, basándose en su mayor estabilidad a la prueba; recordemos que se habla de $S$. aureus meticilino resistente como un estado importante del germen. Sin embargo, nuestros resultados apuntan a que dicha generalidad no debería ser tomada en cuenta, pues, al emplear los dos antibióticos la sensibilidad no fue la misma.

Es necesario que las medidas de vigilancia para el estado de portador de $S$. aureus (sobre todo el meticilino resistente) se tomen tanto a nivel intrahospitalario como comunitario, ya que en estudios realizados en la población general y pacientes de consultorio externo la frecuencia encontrada equivale a más de la quinta parte de la muestra ${ }^{(21,29)}$. Dicha evaluación debe ser periódica, considerando la variación de resistencia a los antimicrobianos y la frecuencia por áreas de servicio ${ }^{(34)}$. Así mismo, el mejor conocimiento del microorganismo y de la etiopatogenia podría ayudar a disminuir el estado de portador ${ }^{(2,39)}$.

EI S. aureus meticilino resistente es considerado un agente nosocomial ${ }^{(11,20,36)}$, por lo que deben tomarse las medidas preventivas necesarias para evitar el estado de portador, la contaminación y transmisión a pacientes $u$ otros trabajadores de salud.

Concluimos que la baja prevalencia de $S$. aureus, hallada en el Hospital Regional de Ica, fue de $10,7 \%$ en manos y de $5,3 \%$ en fosas nasales.

No se observaron diferencias significativas entre las variables estudiadas. Sin embargo, el uso de barreras de protección parece proteger de la contaminación de manos por el S. aureus.

Se encontró una sensibilidad al $100 \%$ a meticilina y vancomicina y resistencia a: oxacilina, dicloxacilina, claritromicina y cloranfenicol en manos; mientras que en fosas nasales no se encontró resistencia a ningún fármaco.

\section{Correspondencia:}

Julissa Huaranga-Bravo

Correo electrónico: shuly_hb@hotmail.com

Célular:955-936217

\section{Agradecimientos.}

A los alumnos de la cátedra de Epidemiología de la Facultad de Medicina de la Universidad Nacional San Luis Gonzaga, quienes amablemente colaboraron en la ejecución de esta investigación.

A los trabajadores del Hospital Regional de Ica por acceder a formar parte de nuestro estudio. 
A la Facultad de Medicina y la Facultad de Ciencias de la Universidad Nacional San Luis Gonzaga, por concedernos sus instalaciones, materiales y equipos para realizar el análisis y procesamiento de las muestras.

\section{REFERENCIAS BIBLIGRÁFICAS}

1 Echevarría J., Iglesias D. Estafilococo Meticilino resistente, un problema actual en la emergencia de resistencia entre los Gram positivos. Rev Med Hered 2003; 14(4):195-214.

2 Hurtado, M, De la Parte M, Brito A. Staphylococcus aureus: Revision of the mechanisms of pathogenicity and physiopathology of staphylococcal infections. Rev. Soc Ven Microbiol 2002; 22(2):1-15.

3 Echevarría J. El problema del Staphylococcus aureus resistente a Meticilina. Rev Med Hered 2010; 21:1-3.

4 Tamariz J, Cruz J, Atencia A, Figueroa J, Horna G, Guerra H. Resistencia a clindamicina inducida por eritromicina en Staphylococcus aureus aislados de tres hospitales de Lima, Perú. Acta Med Per 2009. 26(1):1216.

5 Navarro M. Gestión de riesgo biológico en trabajadores de la salud en centros médicos ambulatorios. Ed. Universidad San Francisco de Quito. Tesis para optar el título de Magíster en Seguridad, Salud y Ambiente. Quito. 2009. Disponible en: http://repositorio.usfq.edu.ec/handle/23000/152

6 Serjan M, Saraceni L. Higiene de manos. Rev Hosp Mat Inf Ramón Sardá 2005. 24(4):158-163.

7 Londoño J, Ortiz G, Gaviria A. Prevalencia de Staphylococcusaureus resistente a meticilina en personal de la unidad de terapia intensiva de la Clínica Universitaria Bolivariana, Medellín 2004. Asociación Colombiana de Infectología. 10(3):161-166. 2006.

8 Castellano M, Bermúdez E, Camacho L. Staphylococcus aureus: Estado de portador en Personal de Enfermería y patrones de susceptibilidad antimicrobiana. Rev Ven Microb 2005; 25(2):1-15.

9 Palacio R, Alonso R, Romero S, Bazet C. Costos de infecciones intrahospitalarias por Staphylococcus aureus; meticilino resistente vs. meticilino sensible. Rev Panam Infectol 2006; 8(1):1-10.

10 Junco R, Marreo M, Lara C. Staphylococcus e Infección Nosocomial. Rev Cubana Hig Epidemiol 2000; 38(1):24-28.

11 Álvarez J, Ramírez A, Mojica-Larrea M, Huerta J, Guerrero J. Staphylococcus aureus resistente a meticilina en un hospital general: panorama epidemiológico del 2000 al 2007. Rev Investigación Clínica 2009; 61(2):98-103.

12 Leal A, Schmalbach J, Álvarez C, Buitrago G, Méndez M. Canales Endémicos y Marcadores de Resistencia Bacteriana, en Instituciones de Tercer Nivel de Bogotá, Colombia. Rev salud público. Sup.; 8(1):59-70.

13 Paz E, Ponce de León D, Ramírez R. Resistencia bacteriana en cuidados intensivos y tendencia actual: Departamento de Cuidados Críticos, Servicio de Cuidados Intensivos del Hospital Nacional Guillermo Almenara Irigoyen, Essalud, Lima, Perú, 2004-2006. Acta Med Per 2008; 25(3):140-147.

14 Hidalgo L, Marroquín J, Antigoni J, Samalvides F. Prevalencia de infecciones hospitalarias en un hospital peruano de nivel IV, en el año 2008. Rev Med Hered $2011 ; 22(2): 76-81$.

15 Heshiki Z., Borsato R., Heshiki R., Matsunaga D., Giampietro L. Flora bacteriana nasal: estudio entre médicos residentes dos Hospitais Universitários de Londrina - Paraná. Ciencias Biológicas e da Saúde; 2002. 23:3-10.

16 Morales R, Batoquio M, Bianchi M, Ducasse E. Detección de portadores nasales de Staphylococcus aureus y su sensibilidad antibiótica. Rev Fac Med UNNE 1996; 13(2):23-27.

17 Morales L, Cedeño J. Frecuencia de Colonización Nasal por Staphyloccus aureus en personal de enfermería de los servicios de medicina interna, cirugía y ginecobstetrícia del Hospital Central Universitario "Dr. Antonio María Pineda" Barquisimeto. Estado Lara. LAPSO 1999-2001. Ed. Universidad Centrooccidental "Lisandro Alvarado". Tesis para optar el título de Especialista en Medicina Interna. Barquisimeto. 2001. Disponible en: http://bibmed.ucla.edu.ve/DB/bmucla/ edocs/textocompleto/TWC195M672001.pdf

18 Guzmán M., Mejía C., Isturiz R., Alvarez C., Bavestrello L. Epidemiology of meticillin-resistant Staphylococcus aureus (MRSA) in Latin America. International Journal of Antimicrobial Agents 2009. 34:304-308.

19 Zelaya L, Zelaya J, Miranda-Soberón U, Guillermo J, Hernández D. Portadores intrahospitalarios de Staphylococcus aureus y sensibilidad a los antimicrobianos. Rev Peru Enf Inf Trop 2001; 1(1):1-6.

20 Mendoza C, Ballón J, De Los Ríos J, Velásquez R. Staphylococcus aureus Meticilino Resistente (MRSA): Colonización y susceptibilidad en pacientes y personal de salud de un hospital de referencia. Diagnóstico. 40(3):1-8. Jun 2001 
21 Platzer L, Aranís C, Beltrán C, Fonseca X, García P. Colonización nasal bacteriana en población sana de la ciudad de Santiago de Chile: ¿Existe portación de Staphylococcus aureus meticilino resistente comunitario?. Rev Otorrinolaringol Cir Cabeza Cuello 2010; 70:109-116.

22 Rodríguez J., Bischofberger C., Álvarez F. Vigilancia y control de Staphylococcus aureus resistente a meticilina en hospitales españoles. Documento deconsenso GEIH-SEIMC y SEMPSPH. Enferm Infecc Microbiol Clin 2008; 26(5):285-298.

23 Osorio L, Hernández E, Fajardo R, Torres E, Mejía G. Eficacia del lavado de manos y alcohol glicerinado en personal de salud. Rev Med IMSS 2004;42(3): 205210.

24 Boyce J, Pittet D. Guía para la Higiene de manos en Centros Sanitarios. CDC, SAED, Morbidity and Mortality Weekly Report 2002;51(16):1-66.

25 Ferrer C. y Almirante B. Higiene de manos: una prioridad para la seguridad de los pacientes hospitalizados. Enferm. Infecc. Microbiol. Clin. 25(6):365-368. 2007.

26 Casanova L, Castañón J. Reflexiones acerca del lavado de manos. Rev Med IMSS 2004;42(6):519-524.

27 Rivera M, Rodríguez C, Huayán G. Frecuencia de aislamientos ambientales de Staphylococcus aureus y su actividad beta-lactamasa en un hospital de Cajamarca, Perú. Revista Infectio 2009;13(3):192-195.

28 Menis A, De Andrade D, Rigotti M, Ferrareza M. Condiciones de limpieza de superficies próximas al paciente en una unidad de terapia intensiva. Rev. Latino Am Enfermagem 2011. 19(3):1-8.

29 Mamani E, Luján D, Pajuelo G. Perfil de sensibilidad y resistencia de Staphylococcus aureus. Experiencia en el Hospital Nacional Hipólito Unanue. An Fac Med Lima 2006. 67(2):120-124.

30 Galí, Z. Profilaxis antimicrobiana: Estrategias en cirugía cardiovascular. Rev Acta Méd 2011; 13(1):101-111.

31 Reyes J, Rincón S, Díaz L, Panesso D, Contreras G. Dissemination of Methicillin-Resistant Staphylococcus aureus USA300 Sequence Type 8 Lineagein Latin. America. CID 2009. 49(12):1-9.

32 Martinez P, Máttar S. Posible aislamiento clínico de Staphylococcus cohnii resistente a vancomicina. Asociación Colombiana de Infectología 2006. 10(3):175-177.

33 Montalvo R., Huaroto L., Alvarezcano J., Ticona E., García Y. Prevalencia de portadores nasales por
Staphylococcus aureus meticilino resistente en personal de salud del servicio de Cuidados intensivos, Hospital Nacional Dos de Mayo. Rev Perú Epidemiol 2009; 13(2):1-5.

34 Maguiña C, Ugarte C, Montiel M. Uso adecuado y racional de los antibióticos. Acta Med Per 2006. 23(1):15-20

35 Loeb M, Main C, Walker-Dilks C, Eady A. Agentes antimicrobianos para erradicar la colonización por Staphylococcus aureus resistente a la meticilina. Reproducción de una revisión Cochrane, traducida y publicada en La Biblioteca Cochrane Plus 2003. (2):126.

36 Mendoza C., Velásquez R., Mercado L., Ballón J., Maguiña C. Susceptibilidad antimicrobiana de Staphylococcus aureus sensible, con sensibilidad "BORDERLINE" y resistentes a la meticilina. Rev Med Hered 2003; 14(4):181-185.

37 Tamariz J., Agapito J., Horna G. y col. Staphylococcus aureus resistente a meticilina adquirido en la comunidad aislados en tres hospitales de LimaPerú. Rev Med Hered 2010; 21:4-10.

38 Callisaya J., Sarmiento Z., Choque H. Prevalencia de Portadores Nasales de Staphylococcus aureus en el personal de limpieza del Hospital Obrero. BIOFARBO $2007 ; 12: 55-60$

39 García C. Staphylococcus aureus meticilino resistente adquirido en la comunidad. Acta Med Per 2011; 28(2):159-162.

Recibido: 10/01/2012

Aceptado para publicación: 24/02/2012 